Int. J. Odontostomat., 6(1):45-50, 2012.

\title{
Displasia Ectodérmica Hipohidrótica - un Síndrome de Interés para la Odontología
}

\author{
Hypohidrotic Ectodermal Dysplasia - a Syndrome of Interest to Dentistry
}

Nilton Alves*; Reinaldo José de Oliveira** \& Naira Figueiredo Deana**

ALVES, N.; OLIVEIRA, R. J. \& DEANA, N. F. Displasia ectodérmica hipohidrótica - un síndrome de interés para la odontología. Int. J. Odontostomat., 6(1):45-50, 2012.

RESUMEN: La displasia ectodérmica hipohidrótica (DEH) es una enfermedad rara de etiología genética. La forma más frecuente es la de herencia recesiva relacionada al cromosomo $X$ con sujetos de sexo masculino afectados y de sexo femenino portadores. Pueden ocurrir a través de mutaciones autosómicas, y en estas, las del gene EDA1 son responsables por la mayoría de los casos. Se caracteriza por la tríada: hipohidrosis, oligodoncia e hipotricosis. Presentamos dos casos de pacientes con DEH en los cuales se observaron signos característicos del síndrome: piel delicada; cabellos, cejas y pestañas escasos; arrugas periorbitales; hiperpigmentación perioral y periorbital; labios prominentes y además de eso el paciente del caso 2 presentaba depresión del puente nasal. Se constató aún disminución de la secreción salival y lagrimal y hipoplasia de maxila en los dos casos. Al examen oral estaban presentes en el caso 1 los caninos superiores derecho e izquierdo temporales y el canino inferior derecho temporal y en el caso 2 los caninos superiores e inferiores (derechos e izquierdos) temporales y dos incisivos superiores (uno derecho y otro izquierdo) permanentes con morfología alterada, siendo todos los elementos dentarios íntegros. El tratamiento odontológico precoz de los portadores de DEH, principalmente en la presencia de oligodoncia, como la que se observa en los casos aquí reportados, es importante no solamente para ofrecer mejor calidad de vida para estos pacientes en corto plazo, sino también en el intento de mitigar las alteraciones en el crecimiento facial a que estos pacientes están sometidos.

PALABRAS CLAVE: displasia ectodérmica hipohidrótica, oligodoncia, síndrome de Christ-Siemens-Touraine.

\section{INTRODUCCIÓN}

Displasias son trastornos del desarrollo de los tejidos y al mismo tiempo las patologías resultantes de ellos. Se clasifican de acuerdo a diversos criterios. En cuanto a los aspectos de malignidad se pueden clasificar en tres grupos: no malignas, premalignas y malignas (Freire-Maia \& Ribeiro, 2002). Según estos autores, las displasias no malignas constituyen la gran mayoría de ellas, incluyendo desde las más simples, tales como manchas y verrugas, hasta las más complejas como los síndromes de displasias y malformaciones. Las displasias premalignas corresponden a conjuntos de células morfológicamente diferenciadas, que no son malignas, pero están predispuestas a la malignización, tales como los pólipos y las lesiones del cuello uterino, producidos por el virus del papiloma humano.
Las displasias malignas se caracterizan por infiltración y metástasis. Estas dos características, asociadas a la capacidad angiogénica, determinan la malignidad (Weinberg, 1998). Las displasias pueden ser puras, cuando ocurren en la forma simple o como un complejo de displasias; sindrómicas, cuando componen el cuadro clínico de los síndromes, o sea, displasias y malformaciones; adquiridas, cuando resultan de la acción de algún factor exógeno, como las radiaciones, por ejemplo, o las heredadas, que siguen un patrón de herencia (Freire-Maia \& Ribeiro).

Displasias ectodérmicas (DE) representan un grupo heterogénico de alteraciones caracterizadas por el desarrollo anormal de derivados embriológicos del

\footnotetext{
* Universidade Estadual Paulista Júlio de Mesquita Filho - UNESP, Araraquara, Brasil.

" Universidade Bandeirante de São Paulo, UNIBAN, Brasil.

${ }^{* * * *}$ Fisioterapeuta, Brasil.
} 
ectodermo. Se han descrito más de 150 tipos (FreireMaia et al., 2001), siendo la forma hipohidrótica la más común.

La displasia ectodérmica hipohidrótica (DEH), descrita por Wedderburn en 1838, también conocida como Síndrome de Christ-Siemens-Touraine, es una enfermedad rara de etiología genética (Neville, 2004). La forma más frecuente es la de herencia recesiva relacionada al cromosoma $X$ con sujetos de sexo masculino afectados y de sexo femenino portadores (Kupietzky \& Houpt, 1995). Puede ocurrir a través de mutaciones autosómicas, y en estas, las del gene EDA1 son responsables del $58 \%$ de los casos (Cluzeau et al., 2011). La DEH presenta tasa de mortalidad infantil entre $2 \%$ y $20 \%$, dependiendo de la precocidad del diagnóstico y de los protocolos de tratamiento (Blüschke et al., 2010).

La DEH es un síndrome de interés para diversas especialidades, incluyendo la Odontología, en especial para la Odontopediatría. Se caracteriza por la tríada: hipohidrosis, oligodoncia e hipotricosis. Otras características suelen estar presentes, tales como los labios inferiores prominentes, história de fiebre, asma y dificultad al respirar, descamación de la piel y pérdida auditiva moderada (Blüschke et al.; Cluzeau et al.).

Aunque no haya cura para la DEH los tratamientos correctivos pueden dar buenos resultados (FreireMaia \& Pinheiro, 1984a,b; Pinheiro \& Freire-Maia, 1991; Freire-Maia, 1999). Entre los profesionales involucrados en estos tratamentos están los odontólogos, de ahí la importancia de que ellos esten familiarizados con este síndrome.

\section{CASO CLÍNICO}

Dos hermanos, sexo masculino, nacionalidad brasileña, con 9 años (caso 1) y 12 años de edad (caso 2) acudieron en clínica privada en la ciudad de São Paulo. En la anamnesis se referió hipertermia de origen desconocida en ambos los casos (hasta los 2 años de edad).

Al examen clínico se observó que ambos presentaban piel delicada y seca; cabellos, cejas y pestañas escasas; arrugas periorbitales; hiperpigmentación perioral; labios prominentes y además, el paciente del caso 2 presentaba depresión del puente nasal (Figs. 1AB y $2 A-B)$. Se constató hipoplasia maxilar y secreción salival y lagrimal disminuidas en ambos casos.

Al examen oral, estaban presentes en el caso 1 los caninos superiores derecho e izquierdo temporales y el canino inferior derecho temporal (Figs. 3A-B); y en el caso 2 los caninos superiores e inferiores (derechos e izquierdos) temporales y dos incisivos superiores (derecho e izquierdo) permanentes con morfología alterada (Figs. 4A-B). Todos los elementos dentários estuvieron íntegros.

Los pacientes fueron sometidos al examen imaginológico para evaluar la presencia de gérmenes dentales, así como, las condiciones en las cuales se encontraban el maxilar y la mandíbula. Ha sido elegida la tomografía computarizada cone-beam, que ofrece imágenes de alta calidad, y además permite reconstrucciones de todas las tomas radiográficas utilizadas en la rutina odontológica (Figs. 5A-B y 6A-B).
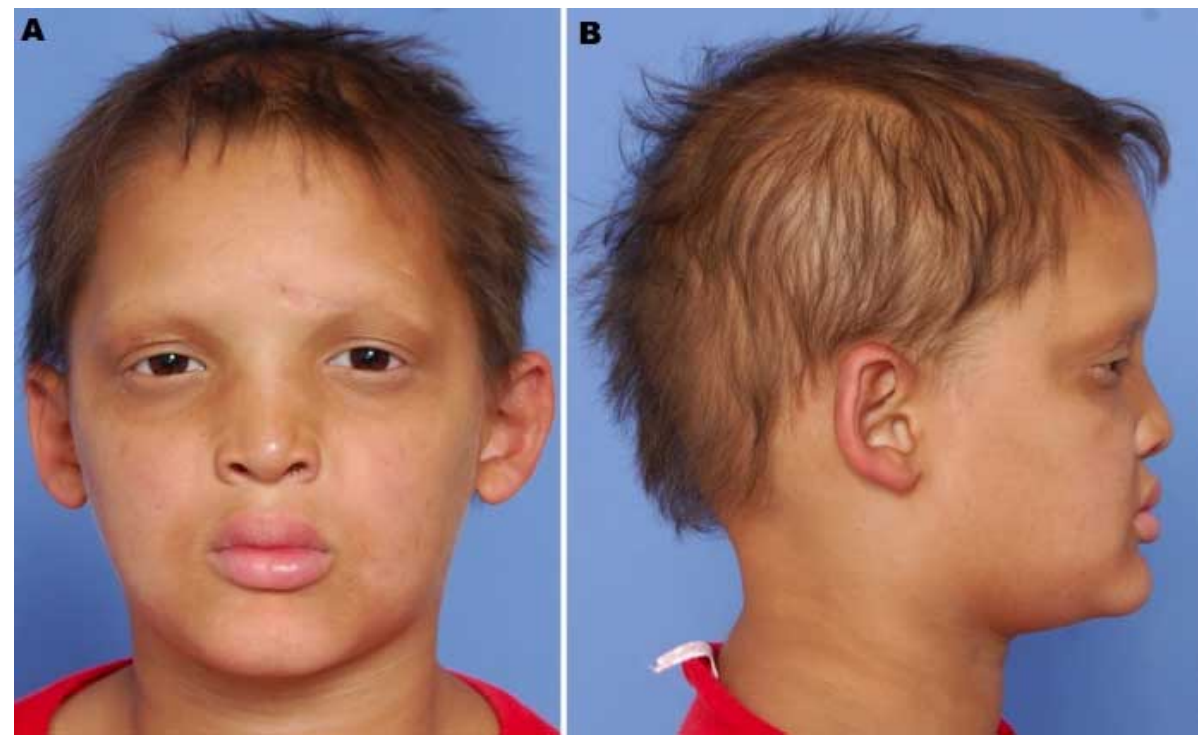

Fig. 1. Paciente caso 1. Signos característicos de DEH. A.- Se puede observar cabellos, cejas y pestañas escasos; arrugas periorbitales: hiperpigmentación perioral y periorbital. B.- Se puede observar labios prominentes. 

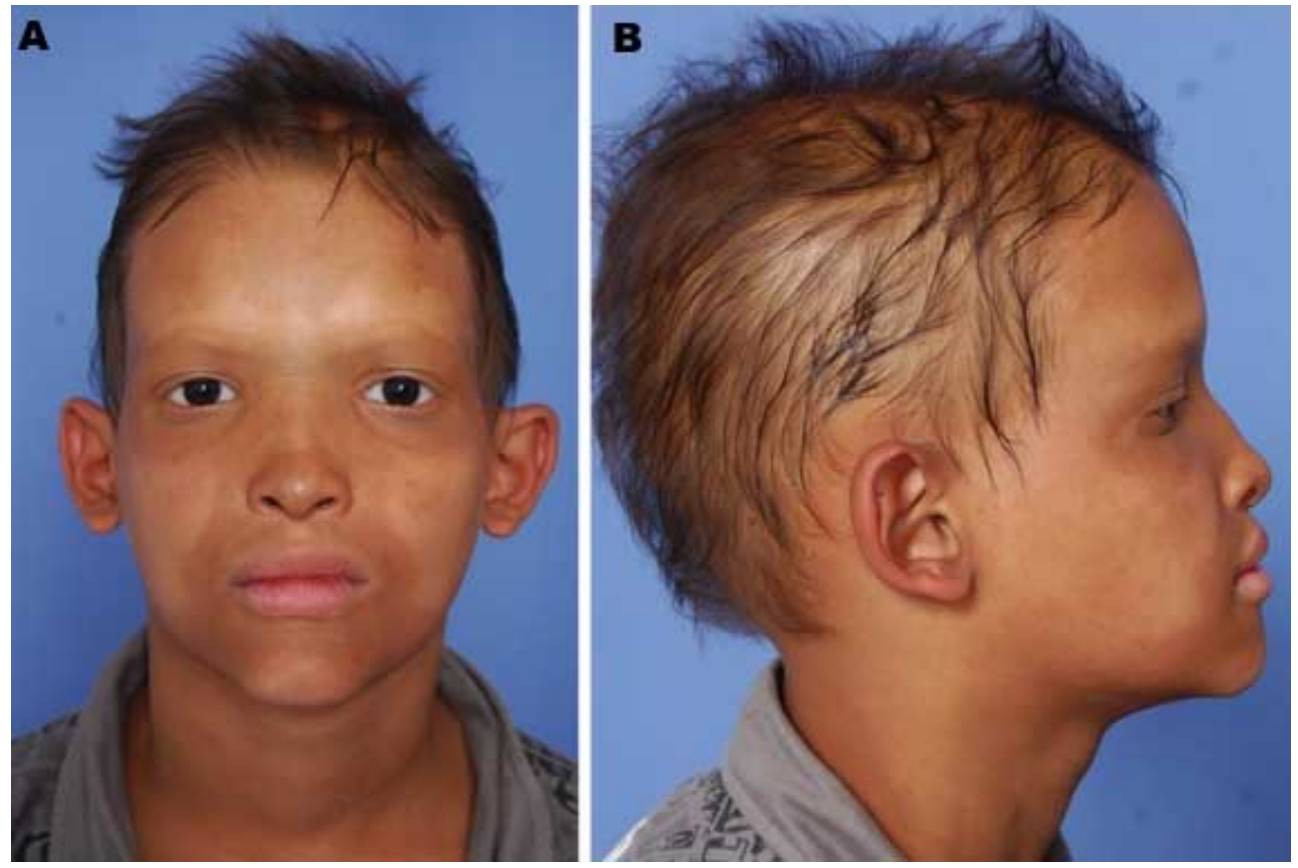

Fig. 2. Paciente caso 2. Signos característicos de DEH. A.- Se puede observar cabellos, cejas y pestañas escasos; arrugas p e r i orbit ales; hiperpigmentación perioral y periorbital. B.Se puede observar labios prominentes y depresión del puente nasal.
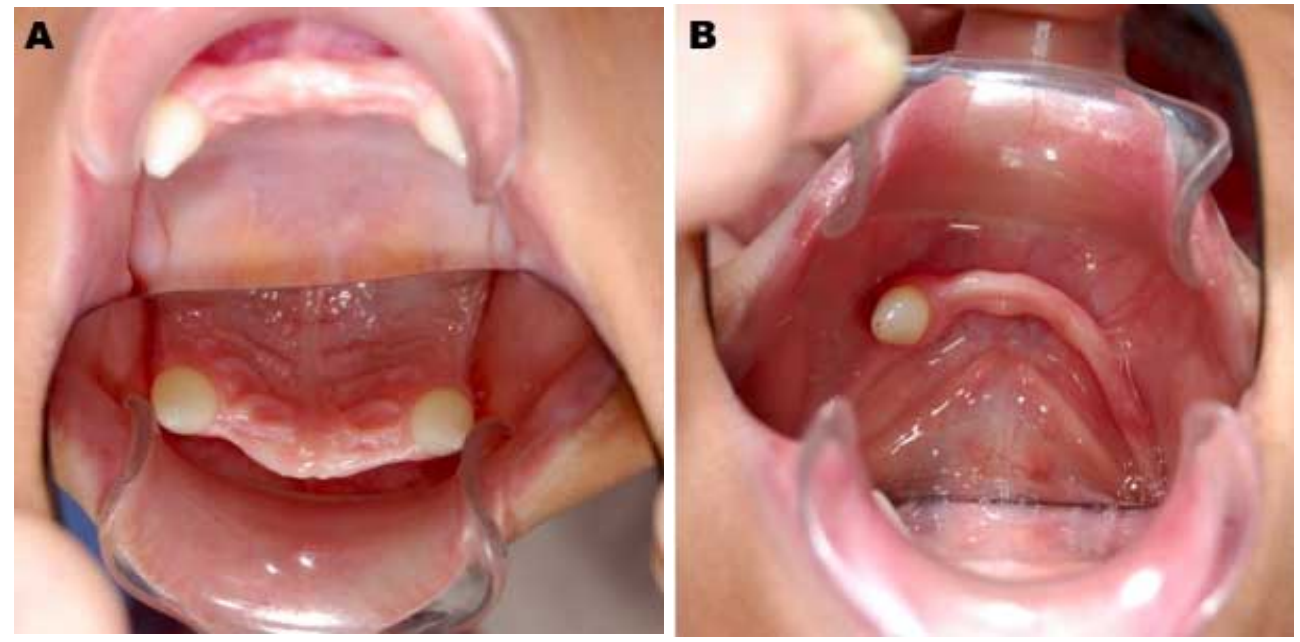

Fig. 3. Paciente caso 1. A.Caninos superiores derecho e izquierdo temporales. B.- Canino inferior derecho temporal.
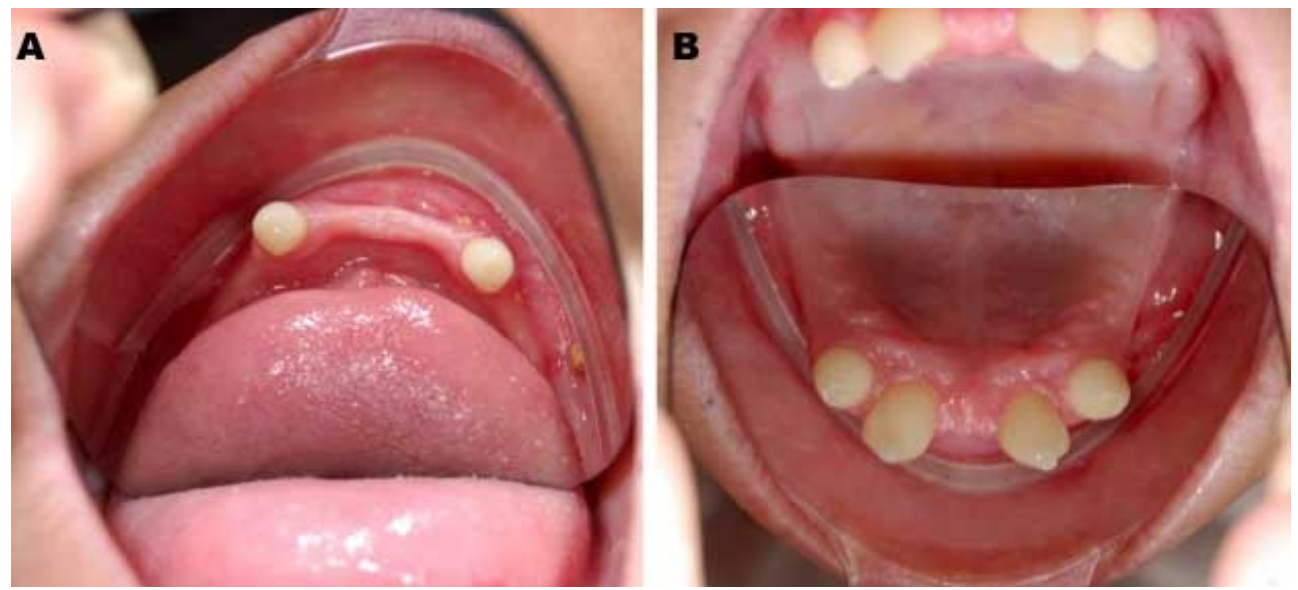

Fig. 4. Paciente caso 2. A.Caninos inferiores derecho e izquierdo temporales. B.Caninos superiores derecho e izquierdo temporales e incisivos superiores derecho e izquierdo permanentes con morfología alterada. 

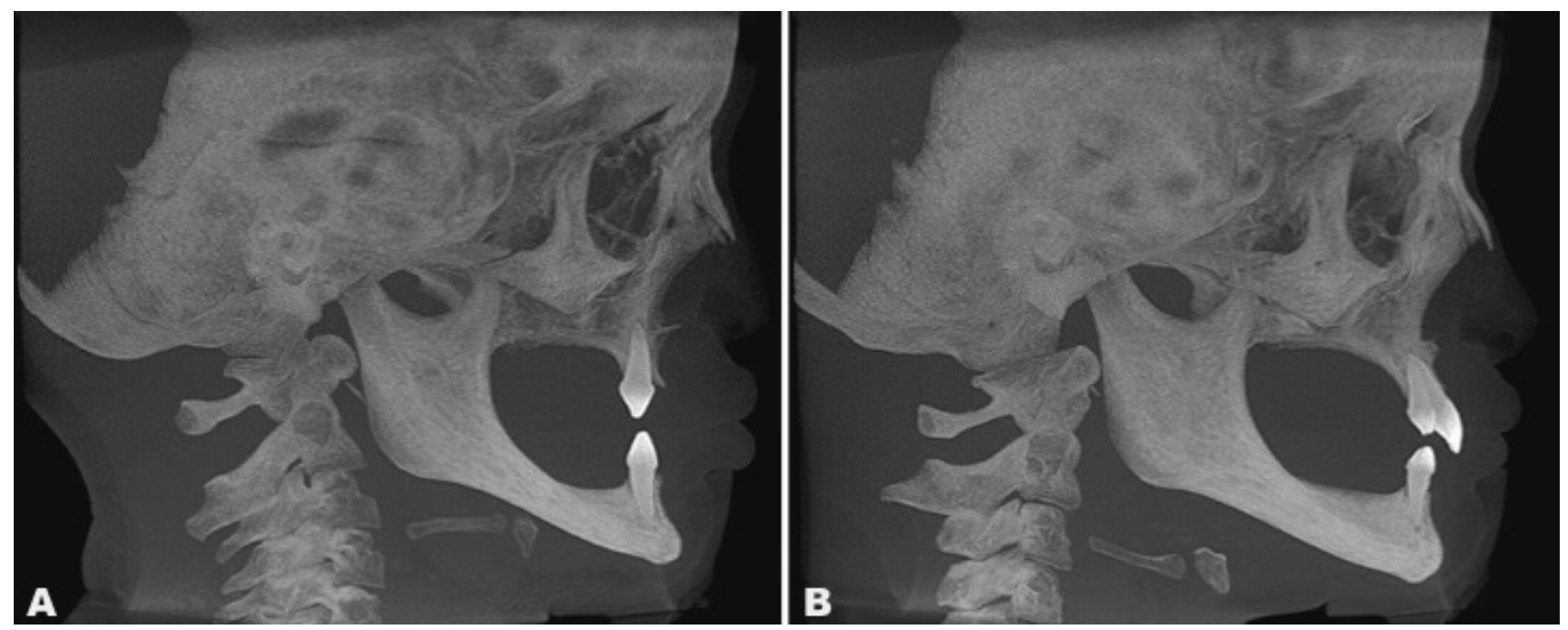

Fig. 5. Imagen de tomografía computarizada volumétrica cone-beam (norma lateral) mostrando ausencia del proceso alveolar en regiones edéntulas de maxila y mandíbula. A.- Paciente caso 1. B.- Paciente caso 2.
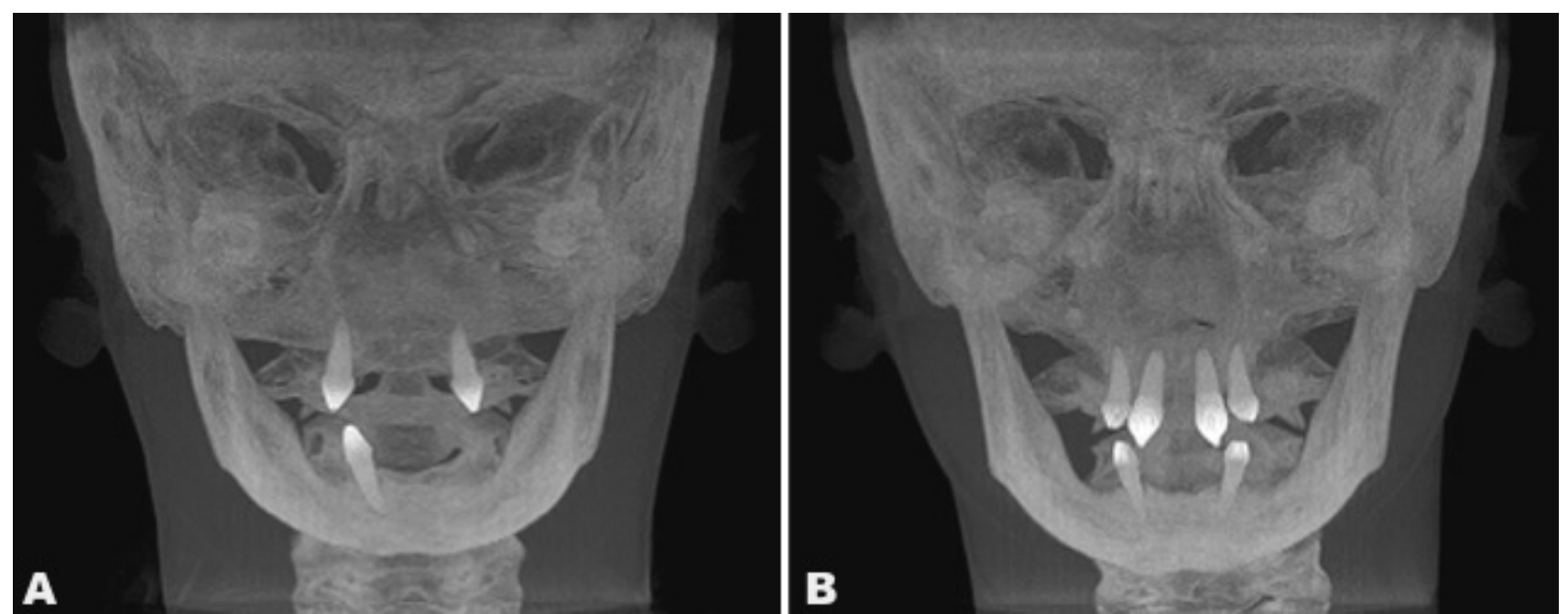

Fig. 6. Imagen de tomografía computarizada volumétrica cone-beam (norma frontal) mostrando oligodoncia e hipoplasia maxilar. A.- Paciente caso 1. B.- Paciente caso 2.

\section{DISCUSIÓN}

La DEH presenta signos característicos, lo que hace su diagnóstico basándose solamente en datos clínicos, relativamente sencillo. En el caso aquí reportado tenemos dos hermanos afectados, lo que hizo aún más seguro el diagnóstico.

Entre los signos característicos la hipohidrosis es lo más grave de ellos, ya que puede determinar fiebre alta con calor, y probablemente esta es la primera razón por la cual la madre busca al pediatra. Junto a eso hay que considerar que la hipohidrosis está pre- sente desde el nacimiento, quizá por eso el pediatra sea el primer profesional de la salud a lo cual se busca (Freire-Maia \& Pinheiro, 1984a, b).

Sin embargo, a veces el pediatra tiene dificultades para hacer el diagnóstico en edad temprana, ya que el paciente además de presentar los dientes no erupcionados, puede tener uñas normales y cabello escaso, o sea, condiciones de normalidad para la edad. Freire-Maia \& Pinheiro (1984a, b) señalaron que en los casos de fiebre alta sin diagnóstico, tratandose del 
paciente de sexo masculino, el pediatra debe comprovar si este niño tiene la "cara diferente". Estos autores sugieren que en estos casos conviene que el pediatra focalice su atención en la cara del paciente, observando por ejemplo: la presencia de hipoplasia maxilar, lábios inferiores prominentes, depresión del puente nasal, hiperpigmentación de la región periorbitaria, entre otras características.

Los odontólogos también son muy buscados para la atención de pacientes portadores de DEH, por lo general en las fases más avanzadas del desarrollo del indivíduo, ya que estos pacientes pueden tener problemas odontológicos importantes.

Con relación específicamente a los problemas de dentición Freire-Maia \& Pinheiro (1984b) describen los más frecuentes: maloclusión, dientes mal posicionados, diastema, microdoncia, supernumerarios, heteromórficos, frágiles, exfoliados, hipocrómicos, con estrias transversales, márgenes irregulares, cáries extensas y precoces, hipoplasia de la corona, cámaras pulpares pequeñas o ausentes, protrusión de los incisivos, incisivos y caninos cuadrangulares, taurodoncia molar, raíces cortas, periodontosis, erosión hasta la encía, displasia de la dentina, manchas marrones, molares piramidales o con raíces fusionadas, anodoncia o hipodoncia (leve, moderada o grave) primaria o secundaria, pseudoanodoncia (con presencia de los gérmenes dentarios de la primera y segunda dentición), persistencia de los dientes temporales, erupción precoz o tardía de los dientes temporales, fusión de los dientes temporales, pérdida temprana o tardía de los dientes temporales, esmalte hipoplásico. Präger et al. (2006) señalan que hipodoncia y malformación suelen ser características dentales regulares en la DEH. Lexner et al. (2007) afirman que la ausencia de dientes permanentes y malformaciones dentales son frecuentes en la $\mathrm{DEH}$, y con relación específicamente a pacientes de sexo femenino heterozigotos existe aumento de la prevalencia de malformaciones dentales además de las dimensiones dentales reducidas, especialmente la mesio-distal.

En los casos aquí descritos observamos que los dos pacientes presentaban oligodoncia y en el caso 2 observamos aún la presencia de dientes heteromórficos. En nuestro trabajo preferimos el término oligodoncia al término hipodoncia, que suele ser utilizado en la literatura, ya que estamos de acuerdo con Kapadia et al. (2007) cuando definen oligodoncia como la ausencia de más de seis dientes y hipodoncia como la ausencia de menos de seis dientes.
Los dientes permanentes comúnmente ausentes en niños portadores de DEH son los incisivos laterales, premolares y segundos y terceros molares. Los primeros molares, incisivos centrales y caninos son los dientes más frecuentemente encontrados (Crawford et al., 1991;. Guckes et al., 1998). Präger et al. afirman que los incisivos laterales superiores son los dientes más comúnmente ausentes, seguidos por los incisivos centrales inferiores. Los dientes más estables son los incisivos centrales superiores, los caninos y primeros molares superiores e inferiores. Los dientes más afectados por alteraciones morfológicas son los incisivos centrales superiores y caninos. Además de eso, estos autores afirman que los dientes caninos temporales y segundos molares temporales suelen ser los más persistentes debido a la agenesia de los incisivos laterales superiores permanentes y segundos premolares inferiores. En el caso 1 de nuestro trabajo observamos la presencia de dos dientes caninos superiores temporales y del canino inferior derecho temporal, y en el caso 2 estaban presentes los caninos superiores e inferiores temporales y dos incisivos superiores permanentes heteromórficos.

Los procesos alveolares que contienen los alvéolos dentarios, se deben a la presencia de los dientes. Su desarrollo depende de la presencia de los elementos dentarios, así comienzan a formarse alrededor de los gérmenes dentarios, y más adelante serán los responsables por el soporte de los dientes, por lo que en su ausencia los procesos alveolares también se quedarán ausentes (Alves \& Cândido, 2008). En ambos los casos aquí reportados notamos la ausencia del proceso alveolar en las regiones edéntulas del maxilar y mandíbula (Figs. 5A-B). Johnson et al. (2002) señalan que en la población con DEH no tratada hubo relación entre el número de dientes permanentes maxilares y la morfología craneofacial, siendo la hipoplasia del tercio medio de la cara y la disminución de la longitud facial las resultantes de la hipodoncia maxilar grave. Esta afirmación está de acuerdo con nuestros hallazgos, ya que observamos en ambos los casos oligodoncia y hipoplasia maxilar (Figs. 6A-B). Se debe recordar que estas alteraciones, además de determinar importantes trastornos funcionales, causan daños psicológicos profundos a los pacientes.

El tratamiento odontológico precoz de los portadores de $\mathrm{DEH}$, principalmente en la presencia de oligodoncia, como la que se observa en los casos aquí reportados, es importante no solamente para ofrecer una mejor calidad de vida para estos pacientes en corto plazo, sino también en el intento de mitigar las alteraciones en el crecimiento facial a que estos pacientes están sometidos. 
ALVES, N.; OLIVEIRA, R. J. \& DEANA, N. F. Hypohidrotic Ectodermal Dysplasia - a syndrome of interest to Dentistry. Int. J. Odontostomat., 6(1):45-50, 2012.

ABSTRACT: Hypohidrotic ectodermal dysplasia (HED) is a rare disease of genetic etiology. The most frequent form is of recessive linked to X-chromosome inheritance with affected male and female carriers. It can occur through autosomal mutations, of the gene EDA1 gene being responsible for the majority of the cases. It is characterized by the triad: hypohidrosis, oligodontia and hypotrichosis. We present two cases of patients with HED in which we observed characteristic signs of this syndrome: delicate skin, sparce hair, eyebrows and eyelashes, periorbital wrinkles, perioral and periorbital hyperpigmentation, prominent lips, in addition the patient in case 2 also present the depressed nasal bridge. We also found decreased salivary and lacrimal secretion and maxillary hypoplasia in both cases. At the oral examination in case 1 the upper right and left deciduous canines and lower right deciduous canine were present, and in case 2 the upper and lower (right and left) deciduous canines and two upper (one right and other left) permanent incisors were present with altered morphology, all of these dental elements were healthy. The early dental treatment of patients with HED, especially in the presence of oligodontia, as observed in our cases, is important not only to provide a better quality of life for these patients in the short term, but also an attempt to minimize the changes in facial growth to which these patients are subject.

KEY WORDS: hypohidrotic ectodermal dysplasia, oligodontia, Christ-Siemens Touraine syndrome.

\section{REFERENCIAS BIBLIOGRÁFICAS}

Alves, N. \& Cândido, P. L. Anatomia para o cirurgião-dentista. 1a ed. São Paulo, Gen-Santos, 2008.

Blüschke, G.; Nüsken, K. D. \& Schneider, H. Prevalence and prevention of severe complications of hypohidrotic ectodermal dysplasia in infancy. Early Hum. Dev., 86(7):3979, 2010.

Cluzeau, C.; Hadj-Rabia, S.; Jambou, M.; Mansour, S.; Guigue, P.; Masmoudi, S.; Bal, E.; Chassaing, N.; Vincent, M.C.; Viot, G.; Clauss, F.; Manière, M. C.; Toupenay, S.; Le Merrer, M.; Lyonnet, S.; Cormier-Daire, V.; Amiel, J.; Faivre, L.; de Prost, Y.; Arnold Munnich, A.; Bonnefont, J. P.; Bodemer, C. \& Smahi, A. Only four genes (EDA1, EDAR, EDARADD, and WNT10A) account for $90 \%$ of hypohidrotic/anhidrotic ectodermal dysplasia cases. Hum. Mutat., 32(1):70-2, 2011.

Crawford, P. J.; Aldred, J. M. \& Clarke, A. Clinical and radiographic dental findings in $\mathrm{X}$ linked hypohidrotic ectodermal dysplasia. J. Med. Genet., 28(3):181-5, 1991.

Freire-Maia, N. \& Pinheiro, M. Ectodermal dysplasias: a clinical and genetic study. New York, Alan R. Liss Inc., 1984a.

Freire-Maia, N. \& Pinheiro, M. Displasias ectodérmicas: manual para profissionais da área de saúde. Curitiba, CEDE, UFPR, 1984b.

Freire-Maia, N. Displasias ectodérmicas: o que são, suas causas, como conviver com elas, onde procurar aconselhamento genético. Curitiba, CEDE, UFPR, 1999.

Freire-Maia, N.; Lisboa-Costa, T. \& Pagan, N. A. Ectodermal dysplasias: how many? Am. J. Med. Genet., 104(1):84, 2001.

Freire-Maia, N. \& Ribeiro, E. M. Dysplasias and malignancies. Am. J. Med. Genet., 113(4):390, 2002.

Guckes, A. D.; Roberts, M. W. \& McCarthy, G. R. Pattern of permanent teeth present in individuals with ectodermal dysplasia and severe hypodontia suggests treatment with dental implants. Pediatr. Dent., 20(4):278-80, 1998.

Johnson, E. L.; Roberts, M. W.; Guckes, A. D.; Bailey, L. J.; Phillips, C. L. \& Wright, J. T. Analysis of craniofacial development in children with hypohidrotic ectodermal dysplasia. Am. J. Med. Genet., 112(4):327-34, 2002.

Kapadia, H.; Mues, G. \& D'Souza, R. Genes affecting tooth morphogenesis. Orthod. Craniofac. Res., 10(3):105-13, 2007.

Kupietzky, A. \& Houpt, M. Hypohidrotic ectodermal dysplasia: characteristics and treatment. Quintessence Int., 26(4):28591, 1995.

Lexner, M. O.; Bardow, A.; Hertz, J. M.; Nielsen, L. A. \& Kreiborg, S. Anomalies of tooth formation in hypohidrotic ectodermal dysplasia. Int. J. Paediatr. Dent., 17(1):10-88, 2007.

Neville, B. W. Patologia Oral e Maxilofacial. 2a ed. Rio de Janeiro, Guanabara Koogan, 2004.

Pinheiro, M. \& Freire-Maia, N. Displasias ectodérmicas. Ciência Hoje, 13(73):56-62, 1991.

Präger, T. M.; Finke, C. \& Miethke, R. R. Dental Findings in patients with ectodermal dysplasia. J. Orofac. Orthop., 67:347-55, 2006.

Weinberg, R. A. One renegade cell: how cancer begins. New York, Basic Books, 1998.

\section{Dirección para Correspondencia}

Dr. Nilton Alves

Rua Humaitá, 1680. CEP 14801-903.

Araraquara - São Paulo

BRASIL

Email: niltonnalves@yahoo.com.br

Recibido : 17-10-2011

Aceptado: 22-12-2011 